and $G$ is the Fourier cosine transform of $g$, then

$$
\int_{0}^{\infty} f(t) g(t) \cos u t d t=\frac{1}{2} \int_{0}^{\infty} F(y)[G(u+y)+G(|u-y|)] d y .
$$

Numerical quadrature of the integral on the left will be laborious if $u$ is large. But, if the transforms $F, G$ are known, the integral on the right may prove much more tractable. The essential reason for this is that the large parameter $u$ now appears in the argument of $G$ as an additive constant and not as a multiplicative constant as in the integral on the left.

As an example, consider the integral

$$
I=\int_{1}^{\infty}\left(t^{2}-1\right)^{-\frac{1}{2}} e^{-a t^{2}} \cos b t d t
$$

for large values of $b$. Here

$$
\begin{aligned}
& f(t)=e^{-a t^{2}} \quad F(u)=\frac{1}{\sqrt{(2 a)}} e^{-u^{2} / 4 a} \\
& g(t)=\left(t^{2}-1\right)^{-\frac{1}{3}} \text { for } t>1 \quad G(u)=-\sqrt{\frac{\pi}{2}} Y_{0}(u) \\
& =0 \quad \text { for } t \leq 1
\end{aligned}
$$

so that

$$
I=-\frac{1}{4} \sqrt{\frac{\pi}{a}} \int_{0}^{\infty} e^{-y^{2} / 4 a}\left[Y_{0}(b+y)+Y_{0}(|b-y|)\right] d y .
$$

This form is, for a wide range of values of $a$ and $b$, very suitable for computation using the simple trapezoidal rule $[1,2]$. The method was used very successfully in preparing a table of an integral, closely related to the one above, which occurs in the theory of the wave resistance of ships [UMT 174, MTAC, v. 7, 1953, p. 248].

This note is published with the permission of the Director of the National Physical Laboratory.

National Physical Laboratory

E. T. Goodwin

Teddington, Middlesex

England

1. E. T. Goodwin, "The evaluation of integrals of the form $\mathcal{S}_{-\infty}{ }^{\infty} f(x) e^{-x^{2}} d x$," Camb. Phil. Soc., Proc., v. 45, 1949, p. 241-245.

2. HENRY E. FETTIS, "Numerical calculation of certain definite integrals by Poisson's Summation Formula," $M T A C$, v. 9, 1955, p. 85-91.

\title{
On a Cubically Convergent Process for Determining the Zeros of Certain Functions
}

An iterative procedure of the form $x_{n+1}=f\left(x_{n}\right)$ for the determination of the roots of the equation $\phi(x)=0$ may be classified as linearly convergent, quadratically convergent, and so on, by using a notation due to Hartree [1]: If $x=X$ 
is a solution of $\phi(x)=0, x_{n}=X+\eta_{n}$ (so that $\eta_{n}$ is the error in the determination of the root at each stage) and $\eta_{n}$ is sufficiently small, then the convergence of the procedure $x_{n+1}=f\left(x_{n}\right)$ is said to be of the $k$-th order if $\eta_{n+1}=0\left(\eta_{n}{ }^{k}\right)$. This means in effect that when using a quadratically convergent procedure we double the number of known figures in the solution at each stage, when using a cubically convergent procedure we treble this number of figures, and so on. An example of a quadratically convergent procedure is provided by the Newton-Raphson process $x_{n+1}=x_{n}-\frac{\phi\left(x_{n}\right)}{\phi^{\prime}\left(x_{n}\right)}$ (where primes denote differentiation with respect to $x$ ). It may be shown that for this process $\lim _{n \rightarrow \infty} \frac{\eta_{n+1}}{\eta_{n}^{2}}=\frac{1}{2} \frac{\phi^{\prime \prime}(X)}{\phi^{\prime}(X)}$, so that the NewtonRaphson process is indeed quadratically convergent.

Various cubically convergent processes for extracting the roots of an equation are known, but they are not generally used because they involve the determination of the second derivative $\phi^{\prime \prime}\left(x_{n}\right)$ and the labor in doing so is normally considered to offset the advantage of gaining the extra figures. It is the purpose of this note to point out that if the function $\phi(x)$ satisfies a differential equation of the form

$$
p \phi^{\prime \prime}+q \phi^{\prime}+r \phi=s
$$

(where $p, q, r$, and $s$ are functions of $x$ ), the determination of $\phi^{\prime \prime}\left(x_{n}\right)$ is a trivial matter, once $\phi\left(x_{n}\right)$ and $\phi^{\prime}\left(x_{n}\right)$ have been calculated. The number of known figures in $X$ may thus be trebled at each stage with scarcely more effort than that necessary to effect the Newton-Raphson process. This remark applies equally well of course if $\phi(x)$ satisfies a nonlinear second order differential equation, but the form of the latter permits of greater variety and hence the subsequent analysis is more complex.

Perhaps the simplest known cubically convergent process is that due to $H$. W. Richmond [2], viz.,

$$
x_{n+1}=x_{n}-\frac{2 \phi\left(x_{n}\right) \phi^{\prime}\left(x_{n}\right)}{2\left(\phi^{\prime}\left(x_{n}\right)\right)^{2}-\phi\left(x_{n}\right) \phi^{\prime \prime}\left(x_{n}\right)} .
$$

It may be shown that for this process $\lim _{n \rightarrow \infty} \frac{\eta_{n+1}}{\eta_{n}{ }^{3}}=\frac{3\left(\phi^{\prime \prime}(X)\right)^{2}-2 \phi^{\prime}(X) \phi^{\prime \prime \prime}(X)}{12\left(\phi^{\prime}(X)\right)^{2}}$

Substituting for $\phi^{\prime \prime}\left(x_{n}\right)$ in (2) from equation (1), we have

$$
x_{n+1}=x_{n}-\frac{2 \phi \phi^{\prime} p}{2\left(\phi^{\prime}\right)^{2} p-\phi s+r \phi^{2}+q \phi \phi^{\prime}},
$$

where the argument $x=x_{n}$ on the right hand side of the equation (3) is understood. An illustration of the power of the method is afforded by the computation of the first three zeros of $J_{0}(x)$, which satisfies the differential equation

$$
x \phi^{\prime \prime}+\phi^{\prime}+x \phi=0,
$$


i.e., $p=x, q=1, r=x, s=0$. For this problem $\phi^{\prime}$ is easily determined from tables since $J_{0}^{\prime}(x)=-J_{1}(x)$. Inspection of the table; [3] shows that zeros of $J_{0}(x)$ occur near $x=2.405, x=5.520$, and $x=8.654$. Table I gives the results obtainable by one application of (3), and contrasts these with those obtained by one application of the Newton-Raphson process.

TABLE I

\begin{tabular}{ccccc}
$\begin{array}{c}\text { Initial } \\
\text { approximation }\end{array}$ & $\begin{array}{c}\text { Result of Newton-Raphson } \\
\text { process }\end{array}$ & \multicolumn{2}{c}{$\begin{array}{c}\text { Result of Richmond's } \\
\text { process }\end{array}$} \\
2.405 & 2.404825551 & 2.404825557697 \\
5.520 & 5.520078110 & 5.520078110286 \\
8.654 & 8.653727909 & 8.653727912914
\end{tabular}

These results were derived solely from tabulated values of $J_{0}(x)$ and $J_{1}(x)$, and required no subsequent interpolation. The quantities in the last column are in error in the last figure by one, one and three units, respectively.

The process is easily extended to the extraction of complex roots. Changing to a new independent variable $z$, where $z=x+i y$, and letting primes now denote differentiation with respect to $z$, we have, after some manipulation

$$
y_{n+1}-y_{n}=\frac{D A-B C}{A^{2}+B^{2}} \quad x_{n+1}-x_{n}=\frac{C A+D B}{A^{2}+B^{2}},
$$

where

$$
\begin{aligned}
& A=2 p_{r e}\left(\phi^{\prime 2}{ }_{r e}-\phi^{\prime 2}{ }_{i m}\right)-4 p_{i m} \phi_{r e}^{\prime} \phi_{i m}^{\prime}-\phi_{r e} s_{r e}+\phi_{i m} s_{i m}+r_{r e}\left(\phi_{r e}^{2}-\phi_{i m}^{2}\right) \\
& -2 r_{i m} \phi_{r e} \phi_{i m}+q_{r e}\left(\phi_{r e} \phi_{r e}^{\prime}-\phi_{i m} \phi_{i m}^{\prime}\right)-q_{i m}\left(\phi_{r e} \phi_{i m}^{\prime}+\phi_{i m} \phi_{r e}^{\prime}\right) \\
& B=4 p_{r e} \phi^{\prime}{ }_{r e} \phi_{i m}^{\prime}+2 p_{i m}\left(\phi^{\prime 2}{ }_{r e}-\phi^{\prime 2}{ }_{i m}\right)-\phi_{i m} s_{r e}-\phi_{r e} s_{i m}+2 r_{r e} \phi_{r e} \phi_{i m} \\
& +r_{i m}\left(\phi_{r e}^{2}-\phi_{i m}^{2}\right)+q_{i m}\left(\phi_{r e} \phi_{r e}^{\prime}-\phi_{i m} \phi_{i m}^{\prime}\right)+q_{r e}\left(\phi_{r e} \phi_{i m}^{\prime}+\phi_{i m} \phi_{r e}^{\prime}\right) \\
& C=2 p_{r e}\left(\phi_{r e} \phi_{r e}^{\prime}-\phi_{i m} \phi_{i m}^{\prime}\right)+2 p_{i m}\left(\phi_{r e} \phi_{i m}^{\prime}+\phi_{i m} \phi_{r e}^{\prime}\right) \\
& D=-2 p_{i m}\left(\phi_{r e} \phi^{\prime}{ }_{r e}-\phi_{i m} \phi_{i m}^{\prime}\right)-2 p_{r e}\left(\phi_{r e} \phi^{\prime}{ }_{i m}+\phi_{i m} \phi_{r e}^{\prime}\right)
\end{aligned}
$$

and the real and imaginary parts of $p\left(z_{n}\right)$ are written $p_{r e}$ and $p_{i m}$, and so on.

An example of the application of the method in the complex case is given by the determination of a zero of $Y_{0}(z)$, which satisfies the differential equation

$$
z \phi^{\prime \prime}+\phi^{\prime}+z \phi=0
$$

i.e., we have $p_{r e}=x_{n}, \quad p_{i m}=y_{n} ; \quad q_{r e}=1, \quad q_{i m}=0 ; \quad r_{r e}=x_{n}, \quad r_{i m}=y_{n}$; $s_{r e}=s_{i m}=0$; and $Y_{0}^{\prime}(z)=-Y_{1}(z)$. Estimation from the tables $[4,5]$ shows that a zero of $Y_{0}(z)$ occurs near $z=-2.3762+i 0.6367$. (The large number of figures in the initial approximation is due to the fact that in these tables $Y_{0}(z)$ is tabulated against the variables $r$ and $\theta$, where $z=r e^{i \theta}$.) Table II displays the results given by the process (4) and contrasts this with the result of one application of the Newton-Raphson process modified for the determination of complex roots, viz.,

$$
x_{n+1}-x_{n}=-\frac{\phi_{r e} \phi_{r e}^{\prime}+\phi_{i m} \phi_{i m}^{\prime}}{\phi_{r e}^{\prime 2}+\phi_{i m}^{\prime 2}} y_{n+1}-y_{n}=\frac{\phi_{r e} \phi_{i m}^{\prime}-\phi_{i m} \phi_{r e}^{\prime},}{{\phi_{r e}^{\prime 2}+{\phi^{\prime 2}}_{i m}}_{r}}
$$


Initial Approximation

$\begin{array}{lr}x & -2.3762 \\ y & 0.6367\end{array}$

\section{TABLE II \\ Newton-Raphson process}

$-2.4047$

0.5407

\author{
Richmond's \\ process
}

0.5398
Correct result

It will be noted that this root is not as accurately determined as were the real roots of $J_{0}(x)=0$. This is not essentially due to the fact that the computations take place in the complex plane, but due to a secondary consequence of this, namely that the initial approximation to the root is more inexact.

Scientific Computing Service Ltd.

\section{P. WYNN}

23, Bedford Square

London, W.C. 1, England

1. D. R. Hartree, "Notes on iterative processes," Cambridge Phil. Soc., Proc., v. 45, 1949, p. 230-236; see also E. SCHRöDER, "Über unendlich viele Algorithmen zür Auflösung der Gleichungen," Math. Ann., v. 2, 1870, p. 317-363. The possible ambiguity in our definition of convergence of order $k$ is not significant in the present context.

2. H. W. RICHMOND, "On certain formulae for numerical approximation," London Math. Soc., Jn., v. 19, 1944, p. 31-38.

3. HaRVARD UnIVERsity, Tables of the Bessel Functions of the First Kind of Orders Zero and One, v. 3, Harvard Univ. Press, Cambridge, 1947.

4. Columbia University, Table of the Bessel Functions $Y_{0}(z)$ and $Y_{1}(z)$ for Complex Arguments, Columbia Univ. Press, New York, 1950.

5. Columbia University, Table of the Bessel Functions $J_{0}(z)$ and $J_{1}(z)$ for Complex Arguments, Columbia Univ. Press, New York, 1943.

\section{REVIEWS AND DESCRIPTIONS OF TABLES AND BOOKS}

33[A].-EDGAR KARST, $A$ simple octal (binary) multiplication method for checking computer results. 4 leaves, handwritten, deposited in the UMT FILE.

In this octal multiplication method, sums of one-digit products are accumulated mentally, with two intermediate lines being written.

\section{J. L. Selfridge}

University of California

Los Angeles, California

34[A-F, HI, K-N, P-V, Z].-KARL SchüTTE, Index Mathematischer Tafelwerke und Tabellen (Index of Mathematical Tables), R. Oldenbourg, München, 1955, 143, p., $21 \mathrm{~cm}$. Canvas cover. Price, DM 14.50.

This is a booklet of 143 pages which consists of the following parts : A list of approximately 1,200 tables and other useful books classified under 16 general headings, an index of authors of these books and tables, and an index of institutes referred to in the first part.

The headings of the 16 classes are as follows: I. Numerical and practical calculating, II. Logarithms of natural numbers, III. Logarithms of circular functions, IV. Natural values of circular functions, V. Simple functions derived from elementary functions, VI. Primes, prime factors, factors, compound interest and rent; continued fractions, theory of numbers, VII. Factorials, gamma functions, exponential and hyperbolic functions; elementary transcendental functions, VIII. 